\title{
How the serotonin transporter 5-HTTLPR polymorphism influences amygdala function: the roles of in vivo serotonin transporter expression and amygdala structure
}

\author{
A Kobiella ${ }^{1,2}$, M Reimold $^{3}$, DE Ulshöfer ${ }^{4}$, VN Ikonomidou ${ }^{5}$, C Vollmert $^{2}$, S Vollstädt-Klein ${ }^{2}$, M Rietschel $^{6}$, G Reischl $^{7}$, A Heinz $^{8}$ \\ and MN Smolka ${ }^{1,9}$
}

The serotonin transporter-linked promoter region (5-HTTLPR) polymorphism of the serotonin transporter gene is associated with amygdala response during negative emotion. The aim of this study was to investigate whether this genotype effect on amygdala function is mediated by current serotonin transporter (5-HTT) levels or rather by genetically induced influences during neurodevelopment, shaping brain structure. A total of 54 healthy subjects underwent functional and structural magnetic resonance imaging, $\left[{ }^{11} \mathrm{C}\right]$ DASB positron emission tomography and 5-HTTLPR genotyping to analyze the interrelationships between amygdala activation during processing of unpleasant stimuli, 5-HTTLPR genotype, amygdala volumes and 5-HTT levels in the midbrain and in other brain regions. In line with previous research, carriers of the short allele (S) showed increased amygdala activation. Path analysis demonstrated that this genotype effect was not procured by current 5-HTT availability but by amygdala structure, with smaller amygdala volumes in the $S$ than in the LL genotype, as well as smaller volumes being associated with increased amygdala activation. Our findings stress the role of genetic effects during neurodevelopment.

Translational Psychiatry (2011) 1, e37; doi:10.1038/tp.2011.29; published online 30 August 2011

\section{Introduction}

Serotonin (5-HT) neurotransmission has a key role in stress sensitivity and in vulnerability to negative affect. ${ }^{1,2}$ The serotonin transporter gene (SLC6A4) encodes the serotonin transporter protein (5-HTT), which acts as a key regulator by removing serotonin from the synaptic cleft. A variation in the promoter region of SLC6A4, serotonin transporter-linked promoter region (5-HTTLPR), influences its transcriptional activity and regulates 5 -HTT expression and density in human cell lines. ${ }^{1,3,4}$ The 5-HTTLPR is a repeat polymorphism with long (L) and short (S) alleles. The $S$ allele is associated with lower 5-HTT expression and function, as well as anxiety and negative mood in healthy individuals. ${ }^{3}$ The recently discovered A/G single-nucleotide polymorphism (rs25531) within the length polymorphism led to the distinction between the variants $L_{A}$ and $L_{G}$, with the latter one found to be functionally similar to the $S$ allele. ${ }^{5,6}$ As the rs $25531 \mathrm{G}$ allele is almost always in phase with the L allele, ${ }^{7} 5$-HTTLPR is often considered a triallelic polymorphism $\left(\mathrm{L}_{\mathrm{A}}, \mathrm{L}_{\mathrm{G}}\right.$ and $\left.\mathrm{S}\right)$.

Besides the intense study of the interplay between the 5-HTTLPR and stress-reactive phenotypes like depression, anxiety and substance use, the study of endophenotypes like neural activation has gained great scientific interest in this context. Previous functional magnetic resonance imaging (fMRI) studies consistently demonstrated that carriers of the $S$ allele show augmented amygdala response to salient environmental stimuli, particularly unpleasant stimuli indicating increased stress sensitivity. ${ }^{8-13}$ So far, however, it is not clear how this genotype effect on amygdala function is mediated. One plausible mechanism could be through in vivo 5-HTT levels (as depicted in the model in Figure 1), which were shown to be negatively associated with amygdala activity. ${ }^{14}$ However, imaging studies investigating the association between 5-HTTLPR and 5-HTT expression in vivo, have revealed inconsistent results. In healthy LL, particularly $L_{A} L_{A}$ carriers, several positron emission tomography (PET) studies using the selective and non-competitively binding 5 -HTT ligand $\left[{ }^{11} \mathrm{C}\right] \mathrm{DASB}$ reported increased 5 -HTT availability in the midbrain, ${ }^{15}$ putamen ${ }^{16}$ and caudate. ${ }^{17}$ Using the tracer $\left[{ }^{123} \mathrm{I}\right] \beta-\mathrm{CIT}$, one single photon emission computed tomography study found increased 5-HTT availability in the midbrain of healthy LL carriers, ${ }^{18}$ which were later all shown to be carriers of two $L_{A}$ alleles. ${ }^{15}$ Another $\left[{ }^{123} \mathrm{I}\right] \beta-\mathrm{CIT}$ single

\footnotetext{
${ }^{1}$ Department of Psychiatry and Psychotherapy, Technische Universität Dresden, Dresden, Germany; ${ }^{2}$ Department of Addictive Behavior and Addiction Medicine, Central Institute of Mental Health, University of Heidelberg, Mannheim, Germany; ${ }^{3}$ Department of Nuclear Medicine, University of Tübingen, Tübingen, Germany; ${ }^{4}$ Department of Psychiatry and Psychotherapy, Central Institute of Mental Health, University of Heidelberg, Mannheim, Germany; ${ }^{5}$ Department of Electrical and Computer Engineering, The Volgenau School of Engineering, George Mason University, Fairfax, VA, USA; ${ }^{6}$ Department of Genetic Epidemiology, Central Institute of Mental Health, University of Heidelberg, Mannheim, Germany; ${ }^{7}$ Department of Preclinical Imaging and Radiopharmacy, University of Tübingen, Tübingen, Germany; ${ }^{8}$ Department of Psychiatry and Psychotherapy, Charité University Medical Center, Campus Mitte, Humboldt Universität Berlin Charité, Berlin, Germany and ${ }^{9}$ Department of Psychology, Neuroimaging Center, Technische Universität Dresden, Dresden, Germany

Correspondence: Professor A Heinz, Department of Psychiatry and Psychotherapy, Charité University Medical Center, Campus Mitte, Humboldt Universität Berlin Charité, Charitéplatz 1, 10117 Berlin, Germany.

E-mail: andreas.heinz@charite.de
}

Keywords: amygdala; fMRI; structural MRI; 5-HTTLPR; 5-HTT; [ $\left.{ }^{11} \mathrm{C}\right] \mathrm{DASB}$ PET

Received 2 March 2011; revised 11 May 2011; accepted 7 July 2011 


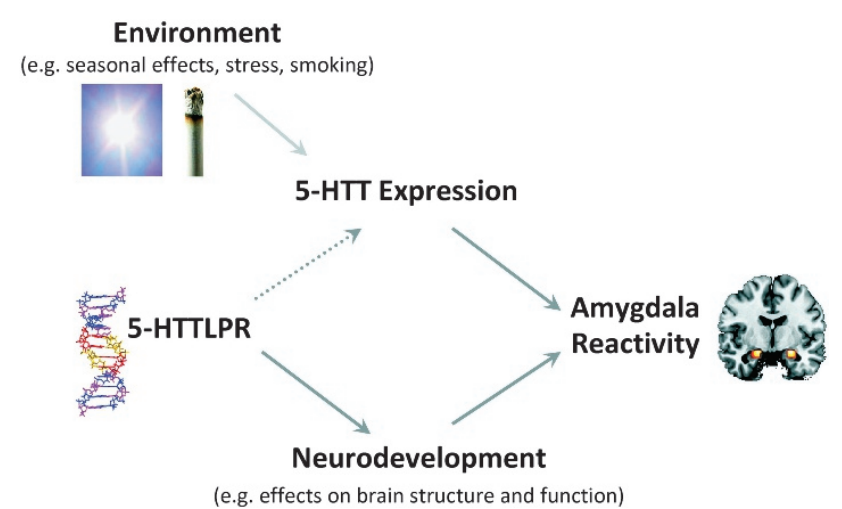

Figure 1 Model of association between 5-HTTLPR, 5-HTT availability and amygdala reactivity. Previous studies indicate that the genotype effect on amygdala reactivity (that is, increased amygdala reactivity in S-allele carriers) is mediated by 5-HTT and/or neurodevelopmentally induced effects on brain structure and function. However, although reduced 5-HTT availability has been associated with increased amygdala activation, findings on the association between 5-HTTLPR and 5-HTT availability have been controversial, as indicated by the dotted line arrow. Inconsistent results might be due to the influence of environmental factors like daylight, stress and smoking on 5-HTT availability.

photon emission computed tomography study reported equal 5-HTT levels in homozygous S- and L-allele carriers, which were increased in comparison with heterozygotes. ${ }^{19}$ Other single photon emission computed tomography and PET studies using the ligands $\left[{ }^{123} \mathrm{I}\right] \mathrm{B}-\mathrm{CIT},\left[{ }^{11} \mathrm{C}\right] \mathrm{McN} 5652$ and $\left[{ }^{11} \mathrm{C}\right] \mathrm{DASB}$ have found no significant association between 5-HTTLPR and 5-HTT. ${ }^{20-24}$ These inconsistencies partly reflect that, beyond the genetic impact, 5-HTT levels are affected by numerous additional factors, including environmental influences like daylight, ${ }^{17,25}$ stress, ${ }^{26}$ alcohol and tobacco, ${ }^{18,27,28}$ and parameters such as sex and age. ${ }^{28-30}$ Furthermore, methodological differences between the studies might explain discrepant results. For example, some radioligands such as $\left[{ }^{123}\right] \beta$-CIT compete with serotonin for binding to $5-\mathrm{HTT}^{31}$, whereas others such as $\left[{ }^{11} \mathrm{C}\right] \mathrm{DASB}$ do not.

In addition or alternatively, the association between the 5-HTTLPR polymorphism and amygdala function could be mediated by genetic effects on neurodevelopment, which shapes the structure and function of brain regions critical for negative emotion processing ${ }^{10,12}$ (Figure 1). In line with this, the genetically driven loss of 5-HTT function in 5-HTT knockout mice led to morphological alterations in the basolateral amygdala. ${ }^{32}$ Healthy S-allele carriers may not only show increased amygdala response to emotional stimuli but also reduced amygdala volume. ${ }^{12,33}$ Smaller amygdala volume and increased amygdala response to emotional stimuli are also observable in unmedicated patients with depression, ${ }^{34-36}$ although those structural alterations do not necessarily drive functional amygdala reactivity. ${ }^{12}$

Our multimodal study aimed to elucidate whether the polymorphic effect of the serotonin transporter gene on amygdala function is mediated by its influence on in vivo 5-HTT and/or amygdala structure. Thus, for the first time, we determined 5-HTTLPR and measured amygdala reactivity and amygdala volumes using $\mathrm{FMRI}$ and structural MRI and in vivo 5 -HTT availability using $\left[{ }^{11} \mathrm{C}\right] \mathrm{DASB}$ PET in the same individuals. We were furthermore interested in the effect of nicotine dependence and therefore investigated both nonsmokers and smokers. We hypothesized that (1) 5-HTTLPR would be associated with 5-HTT levels, with S-allele carriers showing lower 5-HTT levels in vivo, and that this effect would explain the polymorphic effect on amygdala reactivity (increased amygdala activation in S-allele carriers). Consequently, and in line with a previous study, we furthermore expected to find a negative association between 5-HTT levels and amygdala reactivity. ${ }^{14}$ We additionally hypothesized that (2) S-allele carriers would show decreased amygdala volumes and that these structural alterations might furthermore contribute to increased amygdala reactivity. With regard to nicotine dependence, we hypothesized that chronic smoking might elevate 5 -HTT availability, ${ }^{28}$ thereby interfering with the 5-HTT genotype.

\section{Materials and methods}

Participants. A total of 54 healthy individuals were included in the final sample (29 males and 25 females); see below for exclusion criteria. The sample consisted of 29 non-smokers and 26 smokers. Participants were $41.6 \pm 7.9$ (mean \pm s.d.) years of age (range: 30-59). They provided informed, written consent according to the Declaration of Helsinki. The local ethics committee and the Federal Office for Radiation Protection approved the study. Participants were recruited by public announcement.

The Munich-Composite International Diagnostic Interview $^{37}$ was used to assess axis 1 mental disorders. Exclusion criteria were pregnancy, a lifetime history of alcohol and other substance use disorders (except nicotine dependence for smokers), schizophrenia, bipolar disorder or 12 months prevalence of any other axis I disorder. Non-smokers had smoked a maximum of 20 cigarettes in their lives, but none during the past 12 months. All smokers fulfilled the DSM IV (Diagnostic and Statistical Manual of Mental Disorders-IV) criteria for nicotine dependence. They had a Fagerstrøm Test for Nicotine Dependence score of $6.2 \pm 1.7$ (mean \pm s.d.) and had smoked $24.5 \pm 6.6$ (mean \pm s.d.) cigarettes per day during the past 3 months. A urine test for illicit drug use and for pregnancy was applied. Participants were not taking any medication regularly and declared not to have taken any medication and not to have consumed any alcohol 3 days before the experiment. All participants were right handed (laterality quotient in the Edinburgh Handedness Inventory ${ }^{38}$ $>+50$ ) and had a sufficient visual acuity (binocular (corrected) $\geqslant 0.8$.

In all, 12 of the initial 66 participants were excluded from the study because (1) they did not pass thorough screening (and consequently did not undergo brain imaging and blood sampling) $(n=4)$, because of (2) excessive head movement during the functional and/or structural MRI scan $(n=5)$ or because of withdrawn consent in the course of the study $(n=3)$. Non-smokers had participated in another study investigating the effects of nicotine on emotional and motivational processing. ${ }^{39}$ Those participants underwent two fMRI scans within 2 weeks receiving a nicotine gum in 
one session and a placebo gum in the other session in a randomized order. For analysis of the current study, the fMRI data from the placebo session were used. Although we think that it is unlikely that a single dose of nicotine alters 5-HTT availability in a persistent manner, a potential confound of our data could be the administration of a 2-mg nicotine gum in an additional $\mathrm{fMRI}$ session, which preceded the PET scan in 26 non-smokers. In those cases, the time lag between nicotine application and PET scan was $8.4 \pm 5.4$ (mean \pm s.d.) days (range: 3-28 days). Notably, it was not associated with 5-HTT availability in the midbrain $(r=-0.03, P=0.89)$.

The time difference between $\mathrm{fMRI}$ and PET scans was $9.8 \pm 10.5$ (mean \pm s.d.) days (range: $1-52$ days). Participants completed the German version of the state-trait anxiety inventory. $^{40}$ They scored $28.9 \pm 4.7$ (mean \pm s.d.; range: 21-41) on the state anxiety subscale and $30.8 \pm 6.6$ (mean \pm s.d.; range: 20-51) on the trait anxiety subscale.

Genotyping. DNA was isolated from whole blood by standard techniques. 5-HTTLPR variants were determined with PCR techniques. PCR amplification of 5-HTTLPR was as follows: in a total volume of $20 \mu \mathrm{l}, \sim 25 \mathrm{ng}$ of genomic DNA was amplified with the primers as reported previously: ${ }^{7}$ forward 5'-TCCTCCGCTTTGGCGCCTCTTCC$3^{\prime}$ and reverse 5'-TGGGGGTTGCAGGGGAGATCCTG-3' the presence of $1 \times$ Promega PCR Master Mix (Promega Corporation, Madison, WI, USA), without multiplexing. PCR conditions were: $5 \mathrm{~min}$ at $95^{\circ} \mathrm{C}$, followed by 35 cycles of $30 \mathrm{~s}$ at $95^{\circ} \mathrm{C}, 90 \mathrm{~s}$ at $70^{\circ} \mathrm{C}, 60 \mathrm{~s}$ at $72^{\circ} \mathrm{C}$, and a final extension of 10 min at $72^{\circ} \mathrm{C}$. Detection of rs 25531 by restriction digest was performed as follows: $20 \mu$ PCR product was digested with 10 Units Hpall (New England BioLabs, Ipswich, MA, USA) for $4 \mathrm{~h}$ at $37^{\circ} \mathrm{C}$. Samples were run on $3 \%$ agarose gel and precast stained with GelRed (Biotium, Hayward, CA, USA). To score the genotypes, undigested and digested PCR products of the same sample were run on adjacent lanes as described previously. ${ }^{7}$

\section{Functional magnetic resonance imaging}

Task. Affectively unpleasant, pleasant and neutral pictures from the International Affective Picture System ${ }^{41}$ were used to elicit amygdala activation during fMRI. For each category, 40 slides were presented for $2000 \mathrm{~ms}$ in an individually randomized order using an event-related design. The inter-trial interval was randomized between 2.0 and $9.9 \mathrm{~s}$ (mean $6.0 \mathrm{~s}$ ). During the inter-trial interval, a fixation cross was presented. Participants were instructed to passively view the stimuli, as even simple rating tasks can alter brain activation. $^{42}$ To control for attention, a recognition memory test was conducted inside the scanner directly after the scan. The scanning time was $16 \mathrm{~min}$ and $40 \mathrm{~s}$. For further details, see our previous publications. ${ }^{39,43}$

fMRI data acquisition and analysis. Scanning was performed using a 3-T whole-body MRI scanner (Magnetom TRIO; Siemens, Erlangen, Germany) equipped with a standard head coil. For functional imaging, a standard echo planar image $(\mathrm{EPI})$ sequence was used (repetition time $=2410 \mathrm{~ms}$; echo time) $=25 \mathrm{~ms}$; flip angle: $80^{\circ}$ ). fMRI scans were obtained from 42 transversal slices, orientated $30^{\circ}$ clockwise to the anterior commissure-posterior commissure line, with a thickness of $2 \mathrm{~mm}$ (1-mm gap), a field of view of $192 \times 192 \mathrm{~mm}$ and an in-plane resolution of $64 \times 64$ pixels, resulting in a voxel size of $3 \times 3 \times 2 \mathrm{~mm}$. Images were presented through goggles using MRI Audio/Video Systems (Resonance Technology, Northridge, CA, USA). Task presentation was performed using Presentation (Version 9.90, Neurobehavioral Systems, Albany, CA, USA).

Data were analyzed with Statistical Parametric Mapping (SPM5; Wellcome Department of Imaging Neuroscience, London, UK). Before data analysis, functional data underwent preprocessing. The first five images were discarded to reduce T1 saturation effects. Data were temporally realigned with descending slice order to minimize temporal differences in slice acquisition. Spatial realignment was performed to correct for head motion over the course of the session. Functional data were normalized to a standard EPI template, resampled with a voxel size of $2 \times 2 \times 2 \mathrm{~mm}$ and smoothed using an isotropic Gaussian kernel ( $8 \mathrm{~mm}$ full-width at half-maximum).

On an individual level, different conditions (such as pleasant, unpleasant and neutral pictures) were modeled as explanatory variables within the context of the general linear model on a voxel-by-voxel basis. Time derivatives were included in the model to account for temporal differences in the peak of the hemodynamic response.

To interrelate amygdala activation with 5-HTTLPR, 5-HTT and amygdala volume, we extracted the mean signal change from the individual contrast images unpleasant-neutral stimuli applying a bilateral amygdala mask (left amygdala: 161 voxels, right amygdala: 158 voxels) provided by the WFU Pickatlas (Department of Radiology, Wake Forest University School of Medicine, Winston-Salem, NC, USA). To demonstrate that unpleasant versus neutral stimuli elicit significant amygdala activation, we calculated one-sample $t$-tests using SPSS (PASW Statistics 17, SPSS Inc., Chicago, IL, USA). To additionally illustrate the main effects of the task at the whole brain level, we performed a second-level voxel-wise random-effects full factorial analysis with the factor condition (unpleasant, pleasant, neutral). We applied a threshold of $P<0.01$, corrected for family-wise error, with a minimum cluster size of $n=20$ adjacent voxels. For explorative purposes, we also extracted the mean amygdala signal change from the individual contrast images pleasant-neutral stimuli and unpleasant-pleasant stimuli.

Structural MRI data acquisition and analysis. For analysis of brain volume, a three-dimensional T1-weighted magnetization-prepared rapid gradient echo image data set was acquired (repetition time $=1900 \mathrm{~ms}$, echo time $=2.26 \mathrm{~ms}$, field of view $=256 \times 256 \mathrm{~mm}, 176$ slices, $1 \times 1 \times 1 \mathrm{~mm}$ voxel size, flip angle $\left.=9^{\circ}\right)$. The scanning time for the magnetization-prepared rapid gradient echo was $5 \mathrm{~min} 59 \mathrm{~s}$. To measure the volumes of the left and right amygdala, masks of the amygdala structure were created using the automated, model-based, segmentation tool FIRST of FMRIB's Software Library. ${ }^{44,45}$ To account for differences in head sizes, total intracranial volume was measured using FreeSurfer (Martinos Center for Biomedical Imaging, Charlestown, MA, USA) ${ }^{46,47}$ and used as a covariate in the analysis. 


\section{Positron emission tomography}

$P E T$ data acquisition and analysis. $\left[{ }^{11} \mathrm{C}\right] \mathrm{DASB}$ was synthesized as described previously. ${ }^{48}$ After an intravenous bolus injection of $20 \mathrm{mCi}\left[{ }^{11} \mathrm{C}\right] \mathrm{DASB}$, cerebral radioactivity distribution was measured over 90 min using a GE Advance PET scanner (GE Medical Systems, Milwaukee, WI, USA) in two-dimensional acquisition mode. For attenuation correction, a transmission scan with 500.000 kilocounts was used. Three external markers had been attached to the skull to support realignment. Filtered backprojection $(128 \times 128$ pixel $=30 \mathrm{~cm}$ ) with a Hanning filter (cutoff $4.6 \mathrm{~mm}$ ) was used to reconstruct images. Images were realigned and stereotactically normalized with Matlab (Version 7, MathWorks, Sherborn, MA, USA) and SPM2 (Wellcome Department of Imaging Neuroscience). For stereotactic normalization, early summation images $(0-5 \mathrm{~min}$ p.i.) were compared with the standard PET perfusion template provided by SPM. Three-dimensional regions of interest for the midbrain, thalamus, amygdala and cerebellum were taken from previous studies with $\left[{ }^{11} \mathrm{C}\right] \mathrm{DASB} .{ }^{15,26}$ Although the thalamus, amygdala and cerebellum have been defined manually in Montreal Neurological Institute (MNI) space upon anatomical criteria, the midbrain region of interest has been created for $\left[{ }^{11} \mathrm{C}\right] \mathrm{DASB}$ PET studies by applying an isocontour threshold of $\mathrm{BP}_{\mathrm{ND}}>2.5$ to a group of healthy subjects. 5-HTT-binding potential $\mathrm{BP}_{\mathrm{ND}}=\mathrm{k}_{3} / \mathrm{k}_{4}=\mathrm{f}_{\mathrm{ND}} \times \mathrm{B}_{\max } /$ $K_{D}\left(f_{N D}=\right.$ free fraction in the first tissue compartment; $\mathrm{B}_{\max }=$ concentration of binding sites, that is, serotonin transporters; $\mathrm{K}_{\mathrm{D}}=$ dissociation constant) was calculated for each voxel with the multilinear reference tissue model $2 .{ }^{49}$ The washout from the reference tissue $k_{2 R}$, which is required for this analysis, was estimated as described previously. ${ }^{15}$

Statistical analysis. Data were analyzed using SPSS. The threshold of statistical significance was set at $P<0.05$. Those hypotheses which were directed because of consistent findings from previous studies were tested one-tailed; all others two-tailed. We grouped heterozygous and homozygous $S$-allele carriers together $\left(L L=L_{A} L_{A}\right.$; $\mathrm{S}^{\prime}=\mathrm{L}_{\mathrm{A}} \mathrm{L}_{\mathrm{G}} / \mathrm{L}_{\mathrm{A}} \mathrm{S} / \mathrm{SS} / \mathrm{L}_{\mathrm{G}} \mathrm{S} / \mathrm{L}_{\mathrm{G}} \mathrm{L}_{\mathrm{G}}$ ). LL-allele carriers were coded with 0 , and $S$-allele carriers with 1 .

Bivariate correlations were conducted to replicate findings of an association between 5-HTTLPR and amygdala activation to unpleasant stimuli and to display relationships among 5-HTTLPR, midbrain 5-HTT availability, left and right amygdala structures and left and right amygdala activations. Data on amygdala activation elicited by pleasant-neutral stimuli and unpleasant-pleasant stimuli and their associations with 5-HTTLPR, 5-HTT availability and amygdala volume are presented in Supplementary Figure S1 and in Supplementary Table S1. Moreover, state and trait anxiety scores were associated with 5-HTTLPR, 5-HTT availability, amygdala volumes and activations (Supplementary Table S1).

Path analyses using SPSS AMOS 17 (Analysis of Moment Structures; IBM Corporation, Somers, NY, USA) were conducted to test our a priori model of the role of 5-HTT availability and amygdala structure in mediating the relationship between 5-HTTLPR and amygdala activation. The path model (Figure 3) comprised connections between 5-HTTLPR, midbrain 5-HTT availability, left and right amygdala structures and left amygdala activation. In the model, 5-HTTLPR has direct effects on left amygdala activation, bilateral amygdala volumes and midbrain 5-HTT availability and has indirect effects on left amygdala activation through midbrain 5-HTT availability and bilateral amygdala volumes. Moreover, 5-HTT availability and bilateral amygdala volumes have direct effects on left amygdala activation. To further explore the influence of smoking status and to control for the influences of covariates, further analyses were conducted: a multi-group analysis testing for differences between smokers and non-smokers and a path model including the covariates sex, age, intracranial volume and state anxiety. In addition, we calculated a path model including correlated error terms of the left and right amygdala volumes to account for a possible dependency of residual variances (that is, not accounted for by the paths in the model). Significance for all relationships was determined at $P<0.05$; trends were noted at $P<0.10$. Model fits were assessed by $\chi^{2}$ goodness-of-fit tests and by root mean square error of approximations, which are two common indices for deviance of the model from the data. They are presented in Supplementary Information.

To further investigate the relationship between 5-HTTLPR and 5-HTT availability, to test for effects of smoking status on 5-HTT availability and to validate preliminary findings of sex differences in 5-HTT availability and a smoking-specific interaction of those sex differences, ${ }^{28}$ an ANOVA (analysis of variance) was conducted using midbrain $5-\mathrm{HTT}$ availability as a dependent variable, 5-HTTLPR, smoking status and sex as fixed factors and age as covariate.

\section{Results}

Genotype data, 5-HTT availability, amygdala volume and amygdala activation in response to unpleasant stimuli. Triallelic genotyping of 5-HTTLPR revealed that of the 54 participants, $16(29.6 \%)$ were LL, $29(53.7 \%)$ were $S^{\prime} L$ and $9(16.7 \%)$ were $S^{\prime} S^{\prime}\left(L L=L_{A} L_{A} ; S^{\prime} L=S L_{A} / L_{A} L_{G} ;\right.$ $\mathrm{S}^{\prime} \mathrm{S}^{\prime}=\mathrm{SS} / \mathrm{SL}_{\mathrm{G}} / \mathrm{L}_{\mathrm{G}} \mathrm{L}_{\mathrm{G}}$ ). Frequencies did not differ significantly from the Hardy-Weinberg equilibrium $\left(\chi^{2}=0.46 ; P=0.50\right)$. $5-\mathrm{HTT}$ availability was highest in the midbrain, thalamus, amygdala and striatum (Supplementary Figure S2). Midbrain 5-HTT availability was highly associated with thalamic 5-HTT availability $(r=0.84, P<0.001)$ and amygdala 5 -HTT availability $(r=0.67, P<0.001)$. For subsequent analysis, we used midbrain 5-HTT availability. Distributions of thalamic and amygdala 5-HTT availability are reported in Supplementary Table S2.

Magnetic resonance imaging volumetry showed that the size of the left amygdala was $1266.6 \pm 280.5 \mathrm{~mm}^{3}$ (mean \pm s.d.) and that the size of the right amygdala was $1314.8 \pm 330.8 \mathrm{~mm}^{3}$.

The analysis of mean signal change extracted from the bilateral amygdala showed that unpleasant compared with neutral stimuli elicited a significant activation in the right and left amygdala: $0.90 \pm 0.88$ (mean \pm s.d.; $\mathrm{t}(53)=7.53, P=6 \times$ $10^{-10}$ ) and $0.88 \pm 1.10$ (mean \pm s.d.; t $(53)=6.16, P=1 \times 10^{-7}$ ), respectively. The results of the voxel-wise whole-brain analysis are displayed in Figure $2 a$ and in Supplementary Table S3. 
Association between 5-HTTLPR and amygdala activation and the mediating role of 5-HTT availability and amygdala volume. 5-HTTLPR was associated with left amygdala activation in response to unpleasant stimuli ( $r=0.30, P=0.015)$. The association between 5-HTTLPR and right amygdala activation was not significant $(r=0.16$, $P=0.129$, Figure $2 b$ ). Table 1 presents bivariate correlations between variables. Path analysis showed that 5-HTTLPR had direct effects on bilateral amygdala volumes (left amygdala volume: path coefficient $(\beta)=-0.30, P=0.012$; right amygdala volume: $\beta=-0.28, P=0.017$, one-tailed), indicating smaller amygdalae volumes in S-allele carriers (Figure 3). The effect of 5-HTTLPR on midbrain 5-HTT availability was negligible. The path between right amygdala volume and left amygdala activation was significant ( $\beta=-0.30, P=0.010$, one-tailed), indicating that individuals with small right amygdala volumes showed increased left amygdala activation. Notably, the direct effect of 5-HTTLPR on left amygdala activation was no longer significant ( $\beta=0.22, P=0.053$, one-tailed). No further relationships were significant (see Supplementary Table S4 and Supplementary Information for a detailed account of the path coefficients). Accounting for the influences of age and sex on midbrain 5-HTT availability, the influence of intracranial volume on amygdala structure and the influence of state anxiety on amygdala activation in response to unpleasant stimuli did not significantly alter the results (see Supplementary Table S5 and Supplementary Figure S3). Finally, the correlation between the error terms of right and left amygdala volumes neither altered the pattern of results nor significantly improved model fit $\left(\chi^{2}=2.202\right.$, $\mathrm{df}=1, \quad P>0.1)$. Thus, the more parsimonious model presented in Figure 3 is to be preferred.

Association between 5-HTTLPR and 5-HTT availability and influence of smoking status and sex. The ANOVA testing for main effects and interactions of 5-HTTLPR, smoking status and sex in predicting 5-HTT availability revealed no overall differences in midbrain 5 - HTT availability between the $S$ and the LL genotypes. Neither did we find a significant influence of smoking status or sex on midbrain 5-HTT availability. However, there was an interaction between 5-HTTLPR and smoking status $(F(1,45)=4.61$; $P=0.037)$ indicating increased midbrain 5-HTT availability in non-smoking homozygote L-allele carriers compared with smoking homozygote L-allele carriers and decreased midbrain 5-HTT availability in non-smoking S-allele carriers compared with smoking S-allele carriers (Figure 4 and Supplementary Table S2). Notably, we did not find an interaction between smoking status and sex regarding the availability of 5-HTT as reported previously. ${ }^{28}$

Association between 5-HTT availability and amygdala activation in response to unpleasant stimuli. Midbrain 5-HTT availability was not associated with left amygdala activation $(r=-0.001, P=0.496)$. However, it was negatively correlated with right amygdala activation ( $r=-0.25, \quad P=0.037$, one-tailed; partial correlation controlling for age and sex: $r=-0.25, P=0.036$, onetailed, see Supplementary Figure S4). There were no associations between left or right amygdala activation and 5-HTT availability in other brain regions.

\section{Discussion}

To the best of our knowledge, this is the first study that investigated the association between 5-HTTLPR, amygdala activation in response to unpleasant stimuli, amygdala volumes and in vivo 5-HTT availability in the same individuals. We tested whether the frequently reported genotype effect on amygdala function $8,10,11,13$ (that is, increased amygdala activation in S-allele carriers) is mediated by in vivo serotonin transporter (5-HTT) levels and/or by amygdala structure,
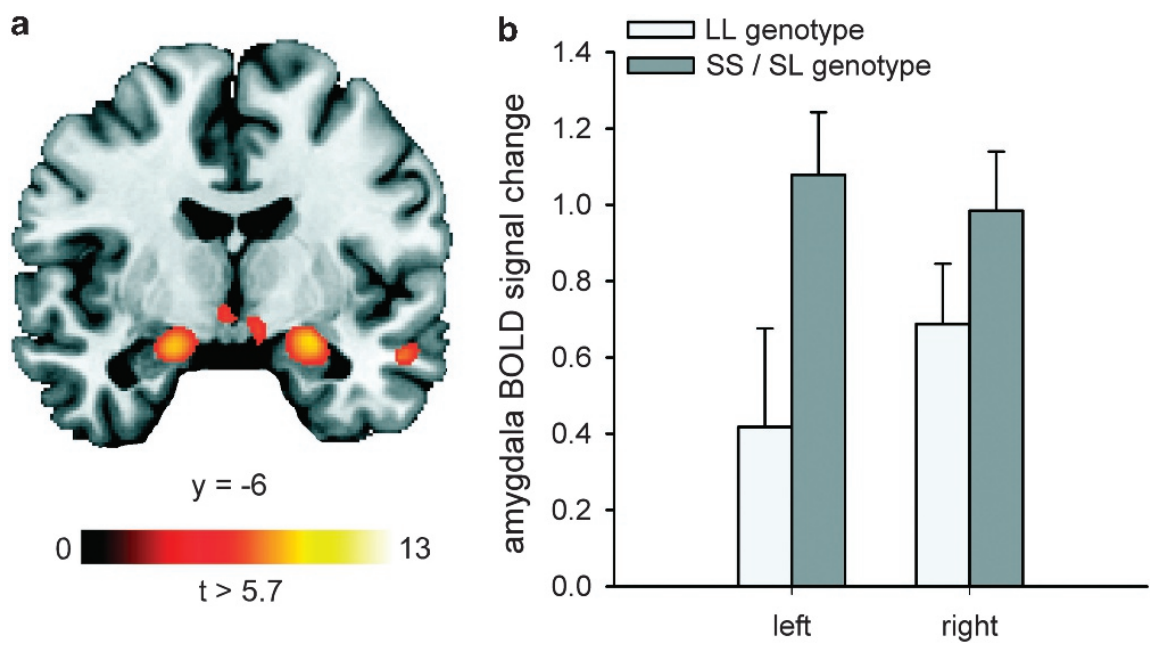

Figure 2 (a) Amygdala activation during presentation of unpleasant compared with neutral stimuli. The statistical parametric map was overlaid on a template T1-weighted magnetic resonance image at $P<0.01$, corrected for family-wise error (FWE). MNI coordinates for the maximal $t$-score in the right and left amygdala are $x=22, y=-6$; $z=-14$ (cluster size: 139 voxels; $t-s c o r e: 9.1$ ) and $x=-20 ; y=-8, z=-16$ (cluster size: 100 voxels; $t$-score: 8.7 ), respectively. (b) Mean left and mean right amygdala response to unpleasant compared with neutral stimuli in the $L L$ and SS/SL genotypes of triallelic 5 -HTTLPR. The $S$ genotype carriers had a higher left amygdala response $(r=0.29, P=0.017)$ but not right amygdala response $(r=0.16 ; P=0.129)$. Error bars indicate +1 standard error, $N=54$. 


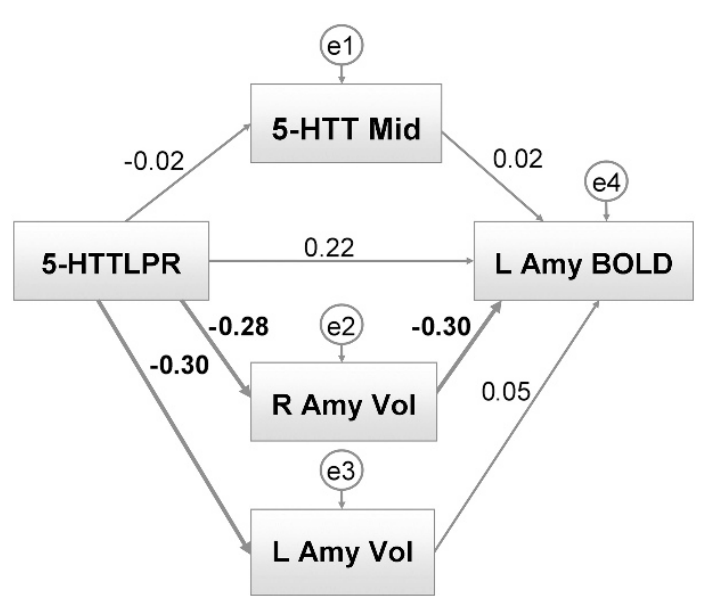

Figure 3 Path analysis predicting left amygdala activation (L Amy BOLD); path coefficients: 5-HTTLPR (triallelic), 5-HTT availability midbrain (5-HTT Mid), left and right amygdala volumes (L Amy Vol, R Amy Vol). Significant paths are depicted in bold lines.

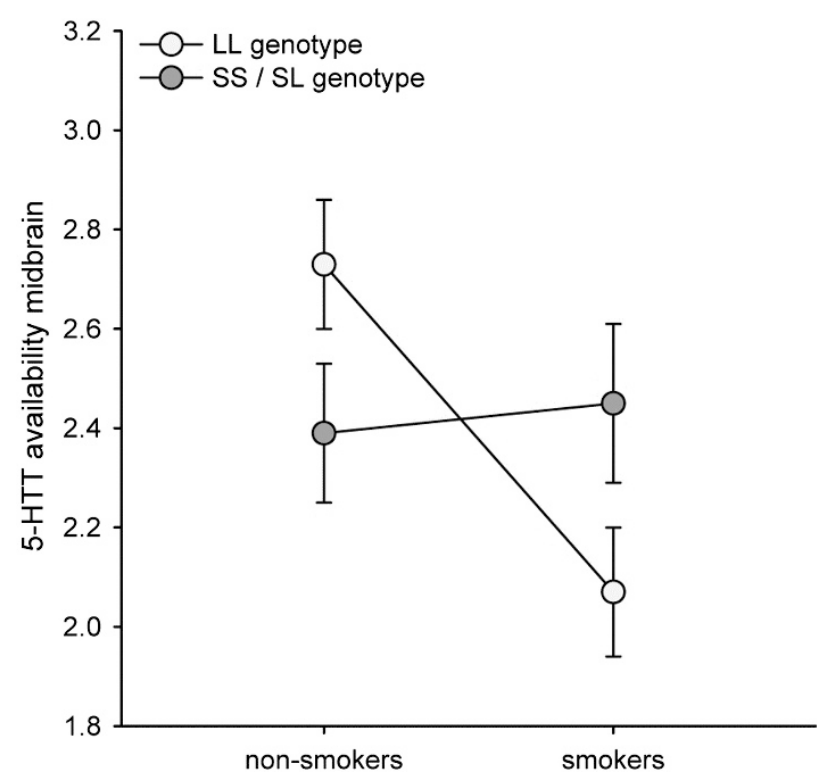

Figure 4 Interaction between 5-HTTLPR and smoking status: non-smoking LL genotype carriers showed increased midbrain 5-HTT levels compared to nonsmoking $S$ genotype carriers, whereas the reverse effect was found in smokers (interaction between 5-HTTLPR and smoking status: $F(1,45)=4.61 ; P=0.037$ ). In the whole group midbrain 5 -HTT availability did not differ between $L L$ and $S$ genotypes. Error bars indicate \pm 1 standard error.

which can be shaped through genetically induced influences during neurodevelopment.

As expected, S-allele carriers showed increased left amygdala activation in response to unpleasant compared with neutral stimuli. Contrary to our hypothesis, this genotype effect on amygdala reactivity, however, could not be explained by direct genotype effects on 5-HTT expression: in the whole group, we found no association between 5-HTTLPR and 5-HTT availability. Neither was 5-HTT availability associated with left amygdala activation. Instead, the polymorphic effect of 5-HTTLPR on amygdala reactivity was mediated by amygdala structure. First of all, S-allele carriers had smaller bilateral amygdala volumes and right 
amygdala volumes predicted left amygdala responses, with smaller volumes being associated with higher activations. Furthermore, in our path model, the direct effect of 5-HTTLPR on amygdala activation was no longer significant. Although decreased amygdala volume and increased amygdala response have been reported in healthy S-allele carriers, as well as in unmedicated patients with depression, $8,10,12,36$ a direct association between amygdala volume and amygdala response to emotional stimuli has so far only been demonstrated in adolescents with bipolar disorder. ${ }^{50}$ In line with our data, the association was inverse in this study. A possible mechanism discussed in the literature and potentially explaining our finding is the effect of 5-HTTLPR polymorphic variation on neurodevelopment, which shapes the structure and function of brain regions critical for negative emotion processing. Thus, it is possible that 5-HTTLPR exerts an early neurodevelopmental influence on amygdala structure, which translates the polymorphic variation into the endophenotype 'amygdala reactivity'. This neurodevelopmental view is supported by studies with 5-HTT knockout mice and rats and with mice and rats prenatally exposed to selective serotonin reuptake inhibitors (SSRI), who both exhibit increased extracellular serotonin levels. ${ }^{51}$ Those studies show that 5-HTT blockade during early development caused alterations in the morphology of pyramidal neurons in the amygdala $^{32}$ and increased anxiety and depression which persisted into adulthood (opposing the effects induced by adult SSRI intake). ${ }^{52,53}$ It is argued that those behavioral and morphological effects are similar to the effects of the S-allele of the 5-HTTLPR polymorphism.

Notably, amygdala volumes did not explain the entire genotype effect on amygdala response to unpleasant stimuli as indicated by the path between 5-HTTLPR and amygdala activation, which showed a weakened (and no longer significant) but not an entirely vanished association. Therefore, it is likely that additional mediators which we did not capture in our model are involved, such as volumes of other brain structures and/or connections between different brain structures.

In line with previous studies, ${ }^{10,13}$ the association between 5-HTTLPR and amygdala function was condition specific: it was present in response to unpleasant compared with neutral stimuli, and not in response to pleasant compared with neutral stimuli (which also elicited pronounced amygdala activation). In contrast to those previous studies and despite a high correlation between left and right amygdala activations ( $r=0.70, \quad P<0.001)$, 5-HTTLPR unexpectedly was not significantly associated with right amygdala activation. Even more surprisingly, we found that right amygdala volume was stronger associated with left amygdala activation than with right amygdala activation. Interestingly, in the non-human and human primate brains, no major interamygdaloid connections have been found. ${ }^{54,55}$ However, functional connectivity is frequently observed in brain regions which are not or only sparsely connected, ${ }^{56}$ pointing to the mediating role of third regions. Therefore, the high correlation of left and right amygdala activations, which is in line with findings from a recent meta-analysis, ${ }^{57}$ could be mediated by a third regionfor example, the prefrontal cortex-which has strong anatomical and functional connections to the amygdala. ${ }^{10,58}$ The mediating influence of a third region might also explain our unexpected finding of an association between right amygdala volume and left amygdala activation. The lack of association between left amygdala volume and left amygdala activation might be due to insufficient power. Future studies will have to replicate our data and clarify the role of laterality.

Interestingly, the association between 5-HTTLPR and 5-HTT availability was modulated by smoking status, with non-smokers replicating previous findings of higher 5-HTT levels in LL genotype carriers compared with S-allele carriers $^{15,16}$ and smokers showing opposite patterns. None of the previous studies which found no association between 5-HTTLPR and 5-HTT availability have reported smoking status of participants, and nicotine abuse or dependence was not an explicit exclusion criterion. Possibly, a converse association between 5-HTTLPR and 5-HTT availability in non-smokers and smokers can lead to a leveling out of whole group effects. Strikingly, a $\left[{ }^{123} \mathrm{I}\right] \beta-\mathrm{CIT}$ single photon emission computed tomography study showed increased midbrain 5-HTT availability in healthy individuals homozygous for the L-allele compared with S-allele carriers and the inverse relationship for alcoholic patients. ${ }^{18}$ This and our present finding indicate a potential role of chronic substance intake in modulating the association between 5-HTTLPR and in vivo 5-HTT availability, which has to be further explored. With regard to the direct effect of nicotine dependence on 5-HTT availability, however, we could not replicate previous findings of increased 5-HTT availability in smokers ${ }^{28}$ as we found no significant differences between smokers and non-smokers.

Our finding of an inverse association between right amygdala activity with unpleasant stimuli and midbrain 5-HTT availability complements findings from a previous study, ${ }^{14}$ although it was less pronounced and not replicated for 5-HTT availability in the amygdala-despite a close association between midbrain and amygdala 5-HTT availability.

A limitation of our study is the relatively small group of homozygous L-allele carriers, which represents the naturally occurring 5-HTTLPR genotype distribution. To increase power, future studies could perform genotyping before assignment to the imaging study to get even sample sizes of L- and S-allele carriers. This would further allow separate analysis of homozygous and heterozygous S-allele carriers.

The fundamental study of the interplay between serotonergic and affective mechanisms in the healthy human brain is a prerequisite for understanding the pathophysiology of mood and anxiety disorders, as well as the effects of pharmacological treatment targeting the serotonergic system. Our multimodal imaging genetic study indicates that the 5HTTLPR genotype effect on amygdala activation is procured by amygdala structure rather than by current effects of 5-HTT availability. Our findings suggest that the path for this genetic effect on emotional reactivity may be paved during neurodevelopment, thereby concurring with animal studies which demonstrate that prenatal SSRI exposure is accompanied by long-term effects on brain structure and by behavioural effects which can dramatically differ from the effects of 5-HTT blockade during adulthood. ${ }^{51}$ Future studies need to investigate the effects of prenatal SSRI exposure on human brain structure and function. Although more than two decades 
have passed since the introduction of the first SSRI, those long-term consequences are completely unknown.

Furthermore, although most previous studies have investigated 5-HTTLPR in relation to amygdala activation or 5-HTT levels, future studies need to highlight the impact of environmental factors in this context and grasp geneenvironment interactions, such as the influence of substances like tobacco and alcohol.

\section{Conflict of interest}

The authors declare no conflict of interest.

Acknowledgements. We thank Juri Rabinstein and Dagmar Gass for help with data acquisition; Jens Treutlein for genotyping; Derik Herman for help with participant information and blood sample taking; Matthias Ruf for technical support; Nils Kroemer for helpful discussion of data analysis; and Karl Mann for general support. This study was supported by the Deutsche Forschungsgemeinschaft (DFG) grants SM 80/2-2, SM 80/5-1, He 2597/7-3 and 4-3 and Re 1472/6-2.

1. Canli T, Lesch KP. Long story short: the serotonin transporter in emotion regulation and social cognition. Nat Neurosci 2007; 10: 1103-1109.

2. Ichise M, Vines DC, Gura T, Anderson GM, Suomi SJ, Higley JD et al. Effects of early life stress on [C-11] DASB positron emission tomography imaging of serotonin transporters in adolescent peer- and mother-reared rhesus monkeys. J Neurosci 2006; 26: 4638-4643.

3. Lesch KP, Bengel D, Heils A, Sabol SZ, Greenberg BD, Petri S et al. Association of anxiety-related traits with a polymorphism in the serotonin transporter gene regulatory region. Science 1996; 274: 1527-1531.

4. Little KY, McLaughlin DP, Zhang L, Livermore CS, Dalack GW, McFinton PR et al. Cocaine, ethanol, and genotype effects on human midbrain serotonin transporter binding sites and mRNA levels. Am J Psychiatry 1998; 155: 207-213.

5. Hu XZ, Lipsky RH, Zhu GS, Akhtar LA, Taubman J, Greenberg BD et al. Serotonin transporter promoter gain-of-function genotypes are linked to obsessive-compulsive disorder. Am J Hum Genet 2006; 78: 815-826.

6. Nakamura M, Ueno S, Sano A, Tanabe H. The human serotonin transporter gene linked polymorphism (5-HTTLPR) shows ten novel allelic variants. Mol Psychiatry 2000; 5: 32-38.

7. Wendland JR, Martin BJ, Kruse MR, Lesch KP, Murphy DL. Simultaneous genotyping of four functional loci of human SLC6A4, with a reappraisal of 5-HTTLPR and rs25531. Mol Psychiatry 2006; 11: 224-226.

8. Hariri AR, Mattay VS, Tessitore A, Kolachana B, Fera F, Goldman D et al. Serotonin transporter genetic variation and the response of the human amygdala. Science 2002; 297: 400-403.

9. Hariri AR, Drabant EM, Munoz KE, Kolachana LS, Mattay VS, Egan MF et al. A susceptibility gene for affective disorders and the response of the human amygdala. Arch Gen Psychiatry 2005; 62: 146-152.

10. Heinz A, Braus DF, Smolka MN, Wrase J, Puls I, Hermann D et al. Amygdala-prefrontal coupling depends on a genetic variation of the serotonin transporter. Nat Neurosci 2005; 8 : 20-21.

11. Munafo MR, Brown SM, Hariri AR. Serotonin transporter (5-HTTLPR) genotype and amygdala activation: a meta-analysis. Biol Psychiatry 2008; 63: 852-857.

12. Pezawas L, Meyer-Lindenberg A, Drabant EM, Verchinski BA, Munoz KE, Kolachana BS et al. 5-HTTLPR polymorphism impacts human cingulate-amygdala interactions: a genetic susceptibility mechanism for depression. Nat Neurosci 2005; 8: 828-834.

13. Smolka MN, Buhler M, Schumann G, Klein S, Hu XZ, Moayer M et al. Gene-gene effects on central processing of aversive stimuli. Mol Psychiatry 2007; 12: 307-317.

14. Rhodes RA, Murthy NV, Dresner MA, Selvaraj S, Stavrakakis N, Babar S et al. Human 5 -HT transporter availability predicts amygdala reactivity in vivo. $J$ Neurosci 2007; 27: 9233-9237.

15. Reimold M, Smolka MN, Schumann G, Zimmer A, Wrase J, Mann $\mathrm{K}$ et al. Midbrain serotonin transporter binding potential measured with [C-11]DASB is affected by serotonin transporter genotype. J Neural Transm 2007; 114: 635-639.

16. Praschak-Rieder N, Kennedy J, Wilson AA, Hussey D, Boovariwala A, Willeit M et al. Novel 5-HTTLPR allele associates with higher serotonin transporter binding in putamen: a [C-11] DASB positron emission tomography study. Biol Psychiatry 2007; 62: 327-331.

17. Kalbitzer J, Erritzoe D, Holst KK, Nielsen FA, Marner L, Lehel S et al. Seasonal changes in brain serotonin transporter binding in short serotonin transporter linked polymorphic regionallele carriers but not in long-allele homozygotes. Biol Psychiatry 2010; 67: 1033-1039.

18. Heinz A, Jones DW, Mazzanti C, Goldman D, Ragan P, Hommer D et al. A relationship between serotonin transporter genotype and in vivo protein expression and alcohol neurotoxicity. Biol Psychiatry 2000; 47: 643-649.
19. Van Dyck CH, Malison RT, Staley JK, Jacobsen LK, Seibyl JP, Laruelle M et al. Central serotonin transporter availability measured with [I-123]beta-CIT SPECT in relation to serotonin transporter genotype. Am J Psychiatry 2004; 161: 525-531.

20. Jacobsen LK, Staley JK, Zoghbi S, Seibyl JP, Kosten TR, Innis RB et al. Prediction of dopamine transporter binding availability by genotype: a preliminary report. $A m \mathrm{~J}$ Psychiatry 2000; 157: 1700-1703.

21. Murthy NV, Selvaraj S, Cowen PJ, Bhagwagar Z, Riedel WJ, Peers P et al. Serotonin transporter polymorphisms (SLC6A4 insertion/deletion and rs25531) do not affect the availability of 5-HTT to [C-11] DASB binding in the living human brain. Neuroimage 2010; 52: 50-54.

22. Parsey RV, Hastings RS, Oquendo MA, Hu XZ, Goldman D, Huang YY et al. Effect of a triallelic functional polymorphism of the serotonin-transporter-linked promoter region on expression of serotonin transporter in the human brain. Am J Psychiatry 2006; 163: 48-51.

23. Shioe K, Ichimya T, Suhara T, Takano A, Sudo Y, Yasuno F et al. No association between genotype of the promoter region of serotonin transporter gene and serotonin transporter binding in human brain measured by PET. Synapse 2003; 48: 184-188.

24. Willeit M, Stastny J, Pirker W, Praschak-Rieder N, Neumeister A, Asenbaum S et al. No evidence for in vivo regulation of midbrain serotonin transporter availability by serotonin transporter promoter gene polymorphism. Biol Psychiatry 2001; 50: 8-12.

25. Praschak-Rieder N, Willeit M, Wilson AA, Houle S, Meyer JH. Seasonal variation in human brain serotonin transporter binding. Arch Gen Psychiatry 2008; 65: 1072-1078.

26. Reimold M, Knobel A, Rapp MA, Batra A, Wiedemann K, Strohle A et al. Central serotonin transporter levels are associated with stress hormone response and anxiety. Psychopharmacology 2011; 213: 563-572.

27. Cosgrove KP, Krantzler E, Frohlich EB, Stiklus S, Pittman B, Tamagnan GD et al. Dopamine and serotonin transporter availability during acute alcohol withdrawal: effects of comorbid tobacco smoking. Neuropsychopharmacology 2009; 34: 2218-2226.

28. Staley JK, Krishnan-Sarin S, Zoghbi S, Tamagnan G, Fujita M, Seibyl JP et al. Sex differences in [1-123]beta-CIT SPECT measures of dopamine and serotonin transporter availability in healthy smokers and nonsmokers. Synapse 2001; 41: 275-284.

29. Staley JK, Sanacora G, Tamagnan G, Maciejewski PK, Malison RT, Berman RM et al. Sex differences in diencephalon serotonin transporter availability in major depression. Biol Psychiatry 2006; 59: 40-47.

30. Van Dyck CH, Malison RT, Seibyl JP, Laruelle M, Klumpp H, Zoghbi SS et al. Age-related decline in central serotonin transporter availability with [I-123]beta-CIT SPECT. Neurobiol Aging 2000; 21: 497-501.

31. Heinz A, Jones DW, Zajicek K, Gorey JG, Juckel G, Higley JD et al. Depletion and restoration of endogenous monoamines affects beta-CIT binding to serotonin but not dopamine transporters in non-human primates. J Neural Transm Suppl 2004; 29-38.

32. Wellman CL, Izquierdo A, Garrett JE, Martin KP, Carroll J, Millstein R et al. Impaired stresscoping and fear extinction and abnormal corticolimbic morphology in serotonin transporter knock-out mice. J Neurosci 2007; 27: 684-691.

33. Frodl T, Koutsouleris N, Bottlender R, Born C, Jager M, Morgenthaler M et al. Reduced gray matter brain volumes are associated with variants of the serotonin transporter gene in major depression. Mol Psychiatry 2008; 13: 1093-1101.

34. Hamilton JP, Siemer M, Gotlib IH. Amygdala volume in major depressive disorder: a metaanalysis of magnetic resonance imaging studies. Mol Psychiatry 2008; 13: 993-1000.

35. Ravindran AV, Smith A, Cameron C, Bhatla R, Cameron I, Georgescu TM et al. Toward a functional neuroanatomy of dysthymia: a functional magnetic resonance imaging study. $J$ Affect Disord 2009; 119: 9-15.

36. Siegle GJ, Thompson W, Carter CS, Steinhauer SR, Thase ME. Increased amygdala and decreased dorsolateral prefrontal BOLD responses in unipolar depression: related and independent features. Biol Psychiatry 2007; 61: 198-209.

37. Wittchen HU, Lachner G, Wunderlich U, Pfister H. Test-retest reliability of the computerized DSM-IV version of the Munich-Composite International Diagnostic Interview (M-CIDI). Soc Psychiatry Psychiatr Epidemiol 1998; 33: 568-578.

38. Oldfield RC. The assessment and analysis of handedness: the Edinburgh inventory. Neuropsychologia 1971; 9: 97-113.

39. Kobiella A, Ulshofer DE, Vollmert C, Vollstadt-Klein S, Buhler M, Esslinger C et al. Nicotine increases neural response to unpleasant stimuli and anxiety in non-smokers. Addict Biol 2011; 16: 285-295.

40. Laux L, Glanzmann P, Schaffner P, Spielberger CD. Das State-Trait- Angstinventar (STAI-G). Weinheim: Beltz 1981.

41. Lang PJ, Bradley MM, Cuthbert BN. The International Affective Picture System (IAPS): Affective Ratings of Pictures and Instruction Manual, Technical Report A-8 University of Florida: Gainesville, FL, 2008.

42. Taylor SF, Phan KL, Decker LR, Liberzon I. Subjective rating of emotionally salient stimuli modulates neural activity. Neuroimage 2003; 18: 650-659.

43. Kobiella A, Vollstadt-Klein S, Buhler M, Graf C, Buchholz HG, Bernow N et al. Human dopamine receptor D2/D3 availability predicts amygdala reactivity to unpleasant stimuli. Hum Brain Mapp 2010; 31: 716-726.

44. Smith SM, Jenkinson M, Woolrich MW, Beckmann CF, Behrens TEJ, Johansen-Berg H et al. Advances in functional and structural MR image analysis and implementation as FSL. Neuroimage 2004; 23: S208-S219.

45. Woolrich MW, Jbabdi S, Patenaude B, Chappell M, Makni S, Behrens T et al. Bayesian analysis of neuroimaging data in FSL. Neuroimage 2009; 45: S173-S186. 
46. Fischl B, Salat DH, Busa E, Albert M, Dieterich M, Haselgrove $C$ et al. Whole brain segmentation: automated labeling of neuroanatomical structures in the human brain Neuron 2002; 33: 341-355.

47. Fischl B, Salat DH, van der Kouwe AJW, Makris N, Segonne F, Quinn BT et al. Sequenceindependent segmentation of magnetic resonance images. Neuroimage 2004; 23: S69-S84.

48. Solbach C, Reischl G, Machulla HJ. Determination of reaction parameters for the synthesis of the serotonin transporter ligand [C-11]DASB: application to a remotely controlled high yield synthesis. Radiochimica Acta 2004; 92: 341-344.

49. Ichise M, Liow JS, Lu JQ, Takano T, Model K, Toyama H et al. Linearized reference tissue parametric Imaging methods: application to [C-11]DASB positron emission tomography studies of the serotonin transporter in human brain. J Cereb Blood Flow Metab 2003; 23 1096-1112.

50. Kalmar JH, Wang F, Chepenik LG, Womer FY, Jones MM, Pittman B et al. Relation between amygdala structure and function in adolescents with bipolar disorder. J Am Acad Child Adolesc Psychiatry 2009; 48: 636-642.

51. Homberg JR, Schubert D, Gaspar P. New perspectives on the neurodevelopmental effects of SSRIs. Trends Pharmacol Sci 2010; 31: 60-65.

52. Ansorge MS, Zhou MM, Lira A, Hen R, Gingrich JA. Early-life blockade of the 5-HT transporter alters emotional behavior in adult mice. Science 2004; 306: 879-881.

53. Popa D, Lena C, Alexandre C, Adrien J. Lasting syndrome of depression produced by reduction in serotonin uptake during postnatal development: evidence from sleep, stress, and behavior. J Neurosci 2008; 28: 3546-3554.
54. Demeter S, Rosene DL, Vanhoesen GW. Fields of origin and pathways of the interhemispheric commissures in the temporal-lobe of macaques. J Comp Neurol 1990; 302: $29-53$.

55. Wilson $\mathrm{CL}$, Isokawa M, Babb TL, Crandall PH, Levesque MF, Engel J. Functional connections in the human temporal-lobe. 2. Evidence for a loss of functional linkage between contralateral limbic structures. Exp Brain Res 1991; 85: 174-187.

56. Damoiseaux JS, Greicius MD. Greater than the sum of its parts: a review of studies combining structural connectivity and resting-state functional connectivity. Brain Struct Funct 2009; 213: 525-533.

57. Robinson JL, Laird AR, Glahn DC, Lovallo WR, Fox PT. Metaanalytic connectivity modeling: delineating the functional connectivity of the human amygdala. Hum Brain Mapp 2010; 31: 173-184.

58. Salzman CD, Fusi S. Emotion, cognition, and mental state representation in amygdala and prefrontal cortex. Ann Rev Neurosci 2010; 33: 173-202.

(c) Translational Psychiatry is an open-access journal published by Nature Publishing Group. This work is licensed under the Creative Commons Attribution-Noncommercial-Share Alike 3.0 Unported License. To view a copy of this license, visit http:// creativecommons.org/licenses/by-nc-sa/3.0/

Supplementary Information accompanies the paper on the Translational Psychiatry website (http://www.nature.com/tp) 